ERNEST $\square R L A N D Q$ LAWRENEE BERKELEY NATIDNAL LAB⿴RATURY

$$
\text { CONF-980794-- }
$$

\section{Electron Transport Uniformity Characterization of CdZnTe using Alpha Particles}

\section{RECEIVED OCT 091998 QS TI}

\author{
M. Amman, J.S. Lee, and P.N. Luke \\ Engineering Division
}

MASTER
DISTRIBUTION GF THIS DOCUMENT IS UNLMATED

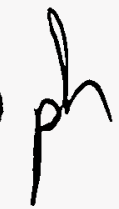

Presented at the

Hard X-Ray and Gamma-Ray Detector

Pbysics and Applications Conference,

San Diego, CA,

July 19-24, 1998,

and to be published in

the Proceedings 


\section{DISCLAIMER}

This document was prepared as an account of work sponsored by the United States Government. While this document is believed to contain correct information, neither the United States Government nor any agency thereof, nor The Regents of the University of California, nor any of their employees, makes any warranty, express or implied, or assumes any legal responsibility for the accuracy, completeness, or usefulness of any information, apparatus, product, or process disclosed, or represents that its use would not infringe privately owned rights. Reference herein to any specific commercial product, process, or service by its trade name, trademark, manufacturer, or otherwise, does not necessarily constitute or imply its endorsement, recommendation, or favoring by the United States Government or any agency thereof, or The Regents of the University of California. The views and opinions of authors expressed herein do not necessarily state or reflect those of the United States Government or any agency thereof, or The Regents of the University of California.

Ernest Orlando Lawrence Berkeley National Laboratory is an equal opportunity employer. 


\section{DISCLAIMER}

Portions of this document may be illegible in electronic image products. Images are produced from the best available original document. 
LBNL-42188

\title{
Electron transport uniformity characterization of CdZnTe using alpha particles
}

\author{
M. Amman, J. S. Lee, and P. N. Luke \\ Engineering Division \\ Engineering Science Department \\ Ernest Orlando Lawrence Berkeley National Laboratory \\ University of California \\ Berkeley, CA 94720
}

April 1998

This work was supported by the Office of Special Technology, Technical Support Working Group (TSWG), of the U.S. Department of Defense under Project No. T-135, through the U.S. Department of Energy under Contract No. DE-AC03-76SF00098. 
Recycled Paper 


\title{
Electron transport uniformity characterization of CdZnTe using alpha particles
}

\author{
M. Amman, J. S. Lee, and P. N. Luke \\ Ernest Orlando Lawrence Berkeley National Laboratory \\ University of California, Berkeley, California 94720
}

\begin{abstract}
Novel electrode configurations, such as coplanar grids, have been successful in mitigating the effects of poor hole transport in CdZnTe gamma-ray detectors. However, poor material uniformity remains a major problem preventing the widespread application of such detectors in gamma-ray spectroscopy. Uniform electron transport is critical for achieving good gamma-ray detection performance in' the coplanar-grid configuration. We have investigated the use of alpha-particle response as a quick and simple electron transport uniformity screening technique for material selection, and as a method to study other spectral broadening mechanisms in coplanar-grid detectors. The method consists of uniformly illuminating, with an alphaparticle source, the cathode side of the $\mathrm{CdZnTe}$ crystal in either a planar or a coplanar-grid detector configuration. In the planar geometry, the variation in the measured pulse heights is dictated in large part by the uniformity of the electron transport. An alpha-particle spectrum that has a single sharp peak with little background indicates uniform electron transport and, consequently, that the CdZnTe crystal should result in a coplanar-grid detector with good gamma-ray detection performance. In the coplanar geometry, the measured pulse-height variation provides information on additional sources of spectral broadening. In this paper we present the results of our study to measure the correlation between these simple alphaparticle measurements and the coplanar-grid gamma-ray detector response.
\end{abstract}

Keywords: gamma-ray detector, coplanar grid, compound semiconductor, $\mathrm{CdZnTe}$, electron transport characterization

\section{INTRODUCTION}

For many radiation detector materials, charge transport deficiencies can be the primary factor limiting the spectroscopic resolution of detectors fabricated from these materials. However, by properly designing the geometry of the detector and the charge-sensing electrodes, the sensitivity to these deficiencies can often be reduced substantially. ${ }^{14}$ This has been clearly demonstrated with the coplanar-grid charge-sensing technique and its variations. ${ }^{s-9}$ With this technique the response of gamma-ray detectors based on CdZnTe has been made substantially insensitive to both the poor hole collection and the significant electron trapping of the material. However, for materials such as $\mathrm{CdZnTe}$, spatial nonuniformity of the material properties remains a barrier to consistently achieving the detector performance necessary for many gamma-ray spectroscopic applications. Electron transport uniformity is a critical property affecting the performance of coplanar-grid $\mathrm{CdZnTe}$ detectors. Certain inhomogeneities affecting the electron transport such as grain boundaries and twins, inclusions, and precipitates can be readily identified using visual inspection, visible light microscopy, and infrared transmission microscopy. ${ }^{10-11}$ Screening the material through these simple methods is unfortunately not sufficient to ensure material uniformity to the extent required for high-resolution gamma-ray spectroscopy applications. Furthermore, no simple method presently exists to efficiently accomplish this material screening task. To address this problem, we have investigated the use of alpha-particle response as a quick, simple, and accurate method to determine the extent of electron transport uniformity in $\mathrm{CdZnTe}$ crystals. Our approach is to form the crystal of interest into a simple planar or a coplanar-grid detector and then to uniformly illuminate the cathode side of the detector with an ${ }^{241} \mathrm{Am}$ alpha-particle source. Each alpha-particle interaction event in the detector generates a well-defined number of electron-hole pairs near the cathode. The collection of this charge results in a detector signal that is almost entirely determined by the transport of the electrons through the entire detector depth. Any spatial variation across the detector in electron transport will be indicated by a variation in the signal pulse heights. Consequently, by uniformly illuminating the cathode side of the detector and measuring the alpha-particle pulseheight spectrum, the extent of electron transport uniformity can be obtained. In this paper we present the results of our alphaparticle response characterization of $\mathrm{CdZnTe}$ material. In particular we demonstrate a correlation between the alpha-particle spectral performance of a crystal in the planar detector geometry to the gamma-ray spectral performance of that same crystal in a coplanar-grid configuration. Following this, the results from alpha-particle measurements made on crystals in the coplanargrid configuration are used to gain information concerning additional spectral broadening mechanisms that contribute to the 


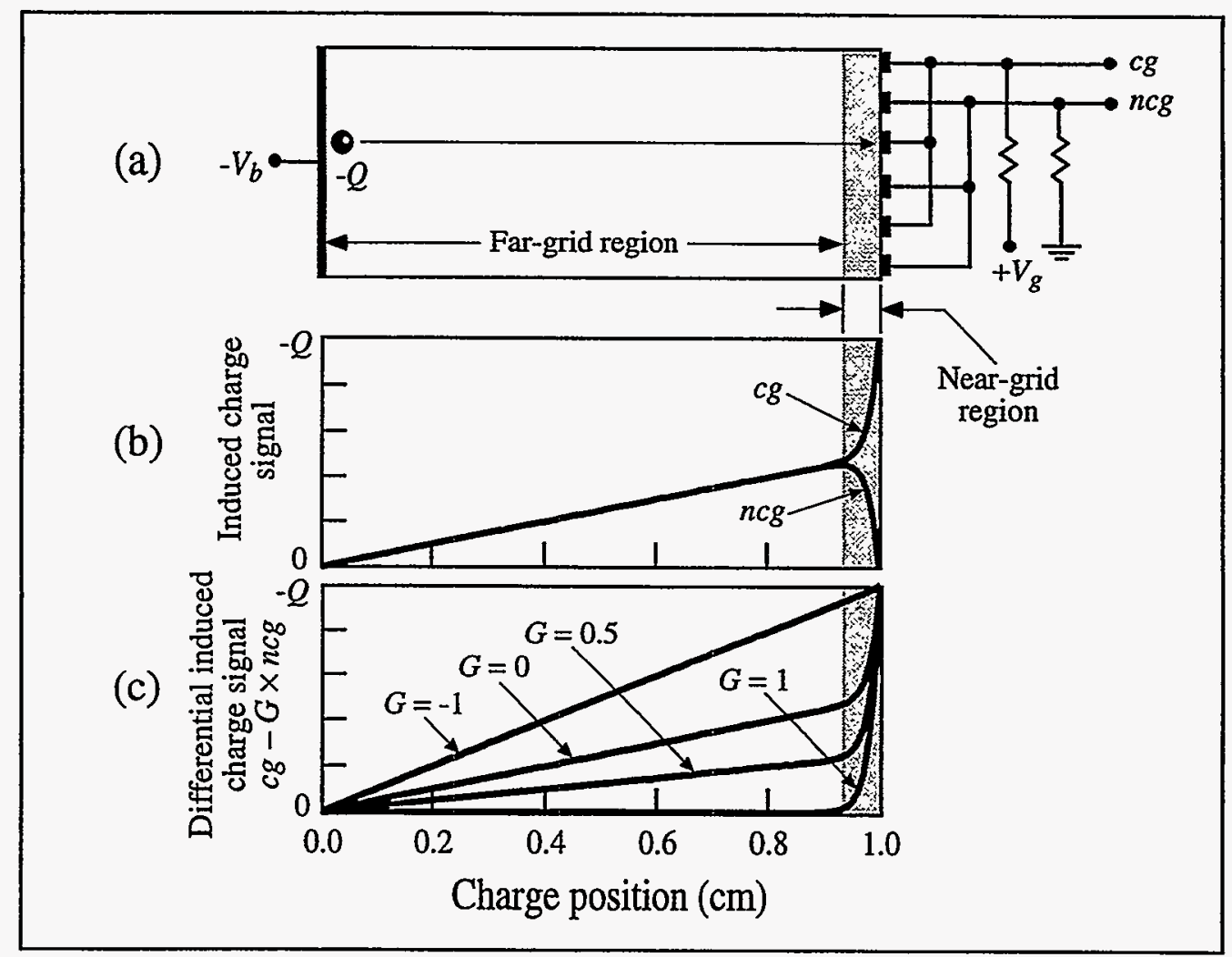

Figure 1. (a) Schematic drawing of a coplanar-grid detector. (b) Calculated induced charge signals at the collecting grid (cg) and noncollecting grid $(n c g)$ of a coplanar-grid detector as a function of the position of a drifting charge $-Q$ originating near the full-area cathode and ultimately collected by the collecting grid as illustrated in (a). The detector is assumed to be $1 \mathrm{~cm}$ thick and infinite in size in the lateral dimensions, and the line width of the grid electrodes is $0.25 \mathrm{~mm}$ with a gap spacing of $0.25 \mathrm{~mm}$. Charge trapping has not been included in the calculation. The region of the detector in which the drifting charge produces the greatest change in the induced charge signals is defined as the near-grid region (shaded region), whereas the remainder of the detector volume is referred to as the far-grid region. (c) Difference between the collecting- and noncollecting-grid signals for various values of the relative gain $G$.

degradation of the gamma-ray detection performance of the detector. We begin with a brief description of the coplanar-grid charge-sensing technique in the following section.

\section{COPLANAR-GRID CHARGE SENSING}

The basic structure and operation of a coplanar-grid detector are illustrated in Figure 1. The detector consists of a radiation sensitive semiconductor crystal with a full-area electrical contact on one detector surface and on the opposing surface a contact segmented into strips that are interconnected to form two interdigitated coplanar grids. A large bias $V_{b}$ is applied between the full contact and the grid electrodes so that the charge carriers created by radiation interactions within the detector crystal are collected across the detector. The bias polarity is such that the electrons drift towards the grid electrodes. A bias $V_{g}$ is also applied between the two grid electrodes to ensure that these electrons are only collected on one of the grids, referred to as the collecting grid. This bias is small relative to $V_{b}$ so that the field within the bulk of the detector remains substantially uniform.

A number of distinct implementations of the coplanar-grid charge-sensing technique exists, ${ }^{6,7,9}$ yet the excellent gamma-ray detection performance achieved with each implementation results from the same two separate aspects of the technique. The first is that the majority of the charge induction occurs when charge carriers drift through a small volume of the detector near the coplanar-grid electrodes (defined as the near-grid region, see Figure 1). This occurs in the conventional coplanar-grid implementation ${ }^{6}$ because charges drifting throughout much of the detector volume (far-grid region) induce the same amount of charge on each grid. Direct subtraction of the two signals produces no net signal in this region (relative gain $G=1$ in Figure 1c). A net detector signal results only when the charge carriers move into the near-grid region causing the 


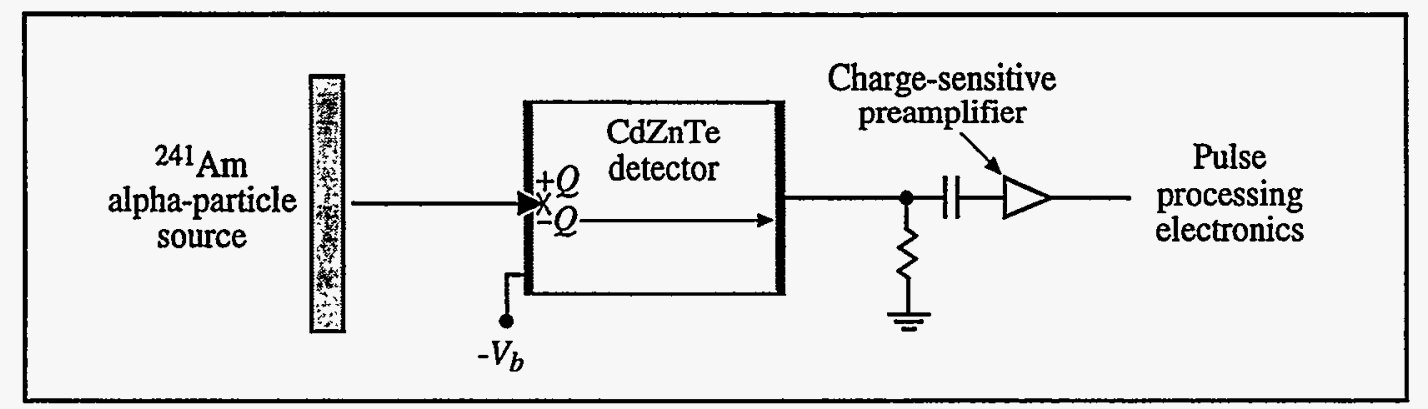

Figure 2. Alpha-particle response measurement configuration for the electron transport uniformity characterization of CdZnTe crystals. In this technique an ${ }^{241} \mathrm{Am}$ alpha-particle source is used to uniformly illuminate the entire cathode side of the CdZnTe crystal in a planar detector geometry. Each alpha particle entering the detector interacts very near the cathode and generates electron-hole pairs. Under the influence of the applied bias $V_{b}$, the generated electrons then drift across the entire depth of the detector. The resultant induced charge pulse on the anode is measured for each event, and its height characterizes the electron transport. Any nonuniformity across the detector in the electron transport is reflected as a variation in the measured pulse heights.

collecting-grid signal to increase at the expense of the noncollecting-grid signal. The collection of electrons through this near-grid region produces a net detector signal that is proportional only to the number of electrons being collected and is therefore unaffected by the poor hole transport for events occurring in the far-grid region.

The second aspect of the coplanar-grid technique that leads to improved spectroscopic performance is that it provides a means to correct for electron trapping. With the conventional coplanar-grid implementation, this is accomplished by adjusting the relative gain $(G)$ of the two grid signals prior to subtraction. This adds a small amount of charge induction in the subtracted detector signal for carriers drifting through the far-grid region (see Figure 1c). By optimizing $G$, compensation can be made for the electron loss during the drift through the far-grid region. In the other coplanar-grid implementations, electron trapping compensation is made by adjusting the relative grid electrode areas, ${ }^{9}$ or by measuring the depth of the gamma-ray interaction and applying a correction to the measured detector signal based on the interaction depth. ${ }^{7}$

In a previous publication, we demonstrated that the effectiveness of the coplanar-grid technique can be compromised by nonuniform charge induction characteristics across the detector, as governed by the detector geometry and grid design. ${ }^{12}$ Another key requirement for the success of the coplanar-grid charge-sensing technique is spatial uniformity in the electron transport. For example, in the conventional coplanar-grid implementation, if the electron trapping varies across the detector, then the optimum value of $G$ to correct for this trapping in one part of the detector is not optimum for another part. This leads to a degradation in the detector resolution. This situation occurs in the other coplanar-grid implementations as well. In addition to electron trapping variation, other factors that influence the electron transport uniformity include electric field uniformity and electron drift length variation. In the following sections we characterize the uniformity of the electron transport in CdZnTe material through alpha-particle response measurements and correlate these results to the gamma-ray detection performance of the material.

\section{ALPHA-PARTICLE SPECTROSCOPY WITH PLANAR GEOMETRY}

Alpha-particle spectroscopy is a simple method to accurately characterize the spatial uniformity of electron transport in CdZnTe crystals. One possible measurement configuration for this technique is shown in Figure 2. The detector crystal to be characterized is first fabricated into a simple planar detector. The fabrication process that we used consists of first mechanically polishing the crystal with an alumina powder slurry to produce smooth surfaces. The surface damage introduced by the mechanical processing is then removed by chemically etching the crystal in an approximately $2 \%$ bromine-methanol solution immediately prior to electrode deposition. The fabrication process is completed by depositing full-area Au electrodes approximately $50 \mathrm{~nm}$ thick on two opposing detector surfaces through thermal evaporation. The completed detector is then mounted into a vacuum chamber where the cathode side of the detector is illuminated with alpha particles from an ${ }^{241} \mathrm{Am}$ source. A thin windowless alpha-particle source is used and the measurements are made under vacuum to ensure that the alpha particles entering the detector have a narrow energy distribution. A bias $V_{b}$ typical of that used for gamma-ray detector operation (1-2 kV for a $1 \mathrm{~cm}$ detector thickness) is applied across the detector to cause the collection of the electrons and holes generated by the alpha-particle interaction events within the detector. Finally the charge 

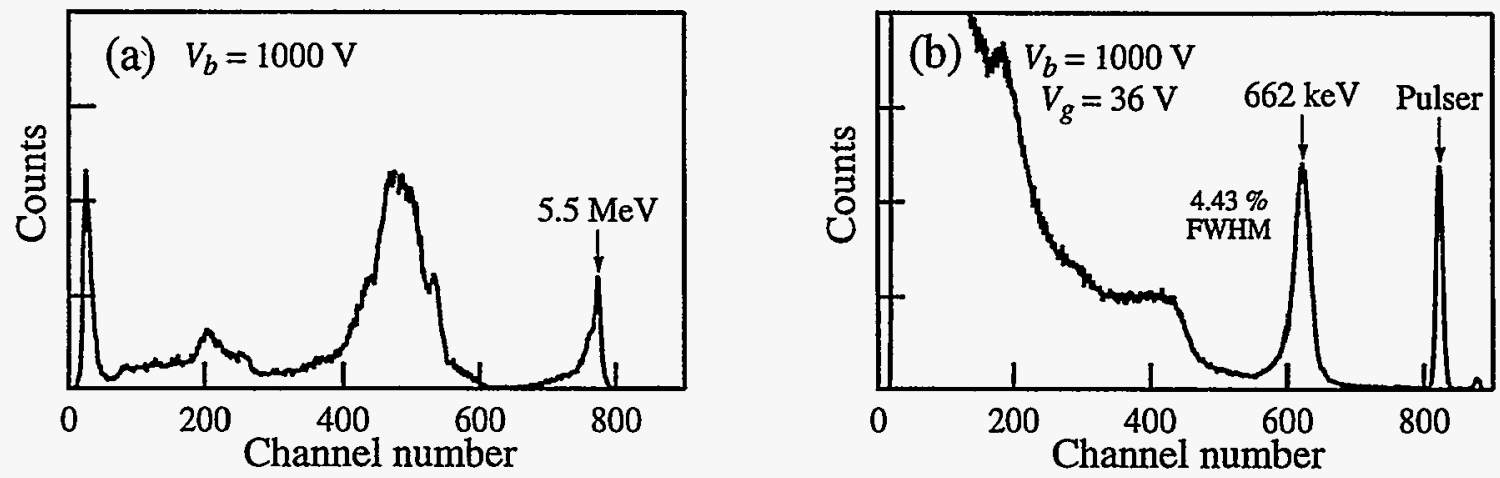

Figure 3. Pulse-height spectra obtained with a $1 \mathrm{~cm}^{3}$ multi-grain CdZnTe detector. (a) Measured ${ }^{24 i}$ Am alpha-particle pulse-height spectrum acquired using a simple planar detector configuration as described in Figure 2 . The detector bias was $1000 \mathrm{~V}$, and the amplifier peaking time was $8 \mu \mathrm{s}$. (b) Measured ${ }^{137} \mathrm{Cs}$ gamma-ray pulse-height spectrum acquired with the same crystal in a conventional coplanar-grid detector configuration. The cathode and grid biases used were $1000 \mathrm{~V}$ and $36 \mathrm{~V}$, respectively, and the amplifier peaking time was $4 \mu \mathrm{s}$. The relative gain $G$ was adjusted in order to produce the best possible $662 \mathrm{keV}$ gamma-ray peak resolution. The $662 \mathrm{keV}$ gamma-ray peak to Compton ratio for this spectrum is 2.4 and was calculated by dividing the number of counts in the peak channel by the average number of counts per channel within the Compton plateau.

pulse induced on the detector anode by the electron-hole pair collection from each alpha-particle interaction event is measured with a charge-sensitive preamplifier and standard pulse processing electronics chain.

Since the alpha particles from the ${ }^{241} \mathrm{Am}$ source (alpha-particle energy of $5.5 \mathrm{MeV}$ ) have a small interaction depth in $\mathrm{CdZnTe}$, all the energy from each particle is deposited within the detector near the entrance contact. Under the influence of the detector bias, the holes generated by each interaction event drift a very short distance before being collected at the cathode, whereas the electrons drift across the entire depth of the detector before being collected at the anode. Consequently, the induced charge pulse on the detector electrodes for each alpha-particle interaction event results almost exclusively from the drift of the electrons. The height of each pulse characterizes the electron transport along the path of the electrons. A variation in the measured pulse heights indicates a variation in the electron transport properties across the detector crystal. Therefore, by measuring a pulse-height spectrum resultant from the full-area cathode illumination with alpha particles, we are able to characterize the uniformity of the electron transport throughout the entire detector volume. An alpha-particle pulse-height spectrum that has a single sharp peak with little background indicates that the detector crystal has the spatially uniform electron transport necessary to produce a coplanar-grid detector with good gamma-ray spectroscopic performance.

We performed the alpha-particle characterization as described above on a number of $1 \mathrm{~cm}^{3} \mathrm{CdZnTe}$ crystals. Then, in order to correlate this alpha-particle performance to the gamma-ray performance in the coplanar-grid configuration, the crystals were fabricated into coplanar-grid detectors for gamma-ray response testing. The fabrication procedure is similar to that of the planar detector, except that the full-area anode is replaced with a contact segmented into strips defined by performing the Au evaporation through a shadow mask. The strip width is $0.25 \mathrm{~mm}$ with a gap of $0.25 \mathrm{~mm}$ between the strips, except near the edge of the detector where the strip widths have been adjusted to minimize edge effects. ${ }^{12}$ The strips are then interconnected with thin Au wire to form the coplanar-grid electrodes. Each completed detector is placed inside a test chamber where it is illuminated with gamma-rays from a ${ }^{137} \mathrm{Cs}$ source and operated as a conventional coplanar-grid detector as described in the previous section. The operating conditions of the detector are then optimized to produce the best possible $662 \mathrm{keV}$ gamma-ray peak resolution.

The results from a CdZnTe crystal with a number of grain boundaries near the cathode side of the detector are shown in Figure 3. The alpha-particle pulse-height spectrum measured using the configuration of Figure 2 contains multiple peaks, a large background, and a relatively small full-energy peak (Figure 3a). This indicates that the electron transport is highly nonuniform in this crystal. The most probable cause of the nonuniformity is trapping at the grain boundaries near the cathode. $^{6}$ The corresponding coplanar-grid gamma-ray performance for this crystal is shown in Figure $3 \mathrm{~b}$. The $662 \mathrm{keV}$ gamma-ray peak resolution has suffered somewhat from the poor electron transport, yet is better than what might initially be expected based on the alpha-particle response. This is because the portion of the crystal with grain boundaries is limited to a small region near the cathode. Detector signals due to gamma-ray interactions outside the region are not affected since the electrons from these interactions never have to pass through the grain boundaries. However, the highly nonuniform transport of the crystal has degraded the gamma-ray detection performance in two additional ways. The background is large as 

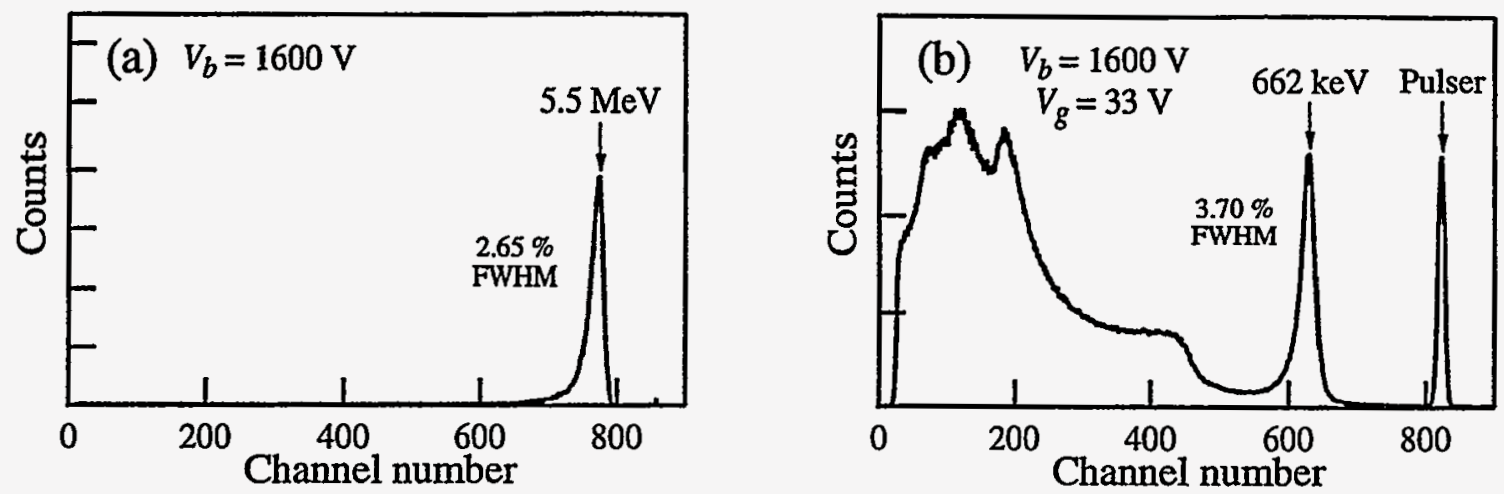

Figure 4. Pulse-height spectra obtained with a $1 \mathrm{~cm}^{3}$ single-crystal CdZnTe detector. (a) Measured ${ }^{241} \mathrm{Am}$ alpha-particle pulseheight spectrum acquired using a simple planar detector configuration as described in Figure 2 . The detector bias was $1600 \mathrm{~V}$, and the amplifier peaking time was $8 \mu \mathrm{s}$. (b) Measured ${ }^{137} \mathrm{Cs}$ gamma-ray pulse-height spectrum acquired with the same crystal in a conventional coplanar-grid detector configuration. The cathode and grid biases used were $1600 \mathrm{~V}$ and $33 \mathrm{~V}$, respectively, and the amplifier peaking time was $4 \mu \mathrm{s}$. The relative gain $G$ was adjusted in order to produce the best possible $662 \mathrm{keV}$ gamma-ray peak resolution. The $662 \mathrm{keV}$ gamma-ray peak to Compton ratio for this spectrum is 3.3 .

evidenced by the high number of counts at low channel numbers, and the photo-peak efficiency is reduced. This is due to the fact that events taking place in the affected region near the cathode induce only a very small signal since most of the electrons are trapped at the grain boundaries before reaching the main charge induction region near the grids.

The results of Figure 3 illustrate the problems that can be introduced when grain boundaries are present in the detector material. However, simply requiring that the detector material be composed of a single crystal is not sufficient to ensure that the electron transport is uniform enough to produce a high spectral resolution in gamma-ray detectors. This is demonstrated with the results of Figure 4 obtained with a single-crystal CdŻnTe detector. The alpha-particle spectrum of Figure $4 \mathrm{a}$ has a single peak with little background, yet the peak width is still quite large, indicating some inhomogeneity in the electron transport. The corresponding coplanar-grid gamma-ray spectrum shown in Figure $4 \mathrm{~b}$ has a lower background and greater photo-peak efficiency than that obtained with the multi-grain detector, but the $662 \mathrm{keV}$ gamma-ray peak resolution is mediocre at $3.7 \%$ FWHM.

Finally, in Figure 5 we show the results from a single-crystal CdZnTe detector with relatively uniform electron transport. The uniformity of the electron transport is indicated by the single sharp peak and little background in the alphaparticle spectrum of Figure 5a. The uniform transport leads to the good coplanar-grid gamma-ray detection performance of
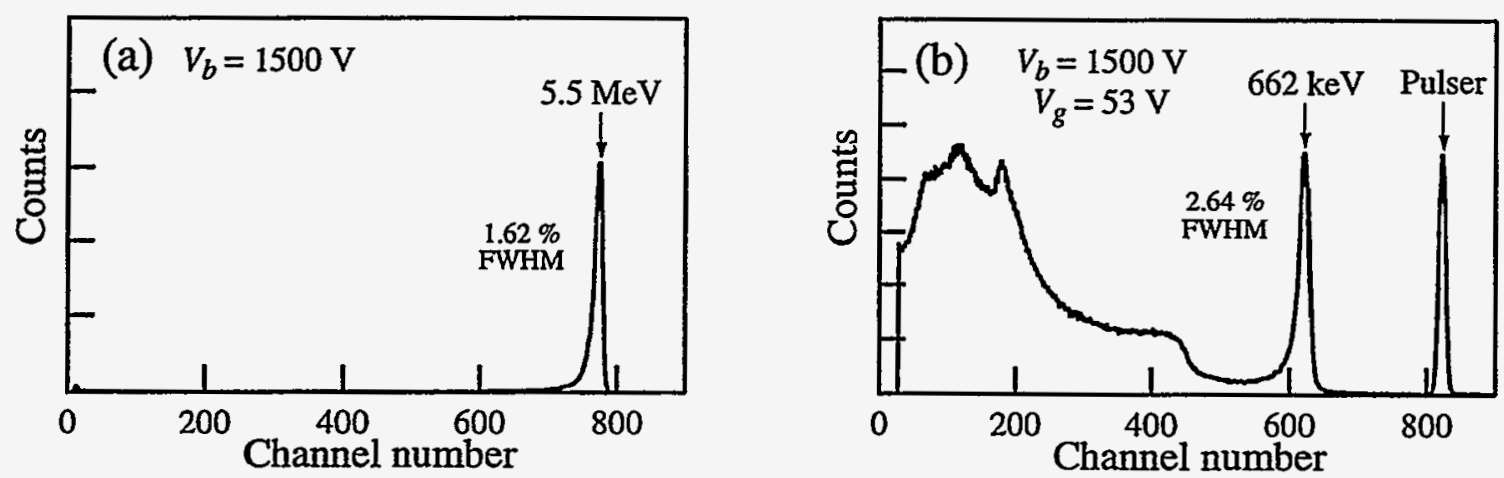

Figure 5. Pulse-height spectra obtained with a $1 \mathrm{~cm}^{3}$ single-crystal CdZnTe detector. (a) Measured ${ }^{241} \mathrm{Am}$ alpha-particle pulseheight spectrum acquired using a simple planar detector configuration as described in Figure 2 . The detector bias was $1500 \mathrm{~V}$, and the amplifier peaking time was $8 \mu \mathrm{s}$. The anode of this detector was actually composed of two grid electrodes, and the grid electrodes were tied together in order to produce a planar detector response. (b) Measured ${ }^{137} \mathrm{Cs}$ gamma-ray pulse-height spectrum acquired with the same crystal in a conventional coplanar-grid detector configuration. The cathode and grid biases used were $1500 \mathrm{~V}$ and $53 \mathrm{~V}$, respectively, and the amplifier peaking time was $8 \mu \mathrm{s}$. The relative gain $G$ was adjusted in order to produce the best possible $662 \mathrm{keV}$ gamma-ray peak resolution. The $662 \mathrm{keV}$ gamma-ray peak to Compton ratio for this spectrum is 4. 
Figure 5b.

These examples demonstrate a good correlation of the alpha-particle spectral response of a CdZnTe crystal in a planar detector geometry to the gamma-ray spectral response of the same crystal using the coplanar-grid charge-sensing technique. Based on these and other results we have obtained, we feel that this simple alpha-particle measurement can be used as a quick and accurate method to screen CdZnTe material based on electron transport uniformity.

\section{ALPHA-PARTICLE SPECTROSCOPY WITH COPLANAR-GRID GEOMETRY}

The resolution of the alpha-particle peak measured using the coplanar-grid charge-sensing technique typically is worse than that measured with the same detector crystal with full-area contacts. A number of mechanisms can lead to this additional broadening including: increased sensitivity to electron transport nonuniformity and trapping, electron collection outside the collecting grid, poor electron collection due to weak field regions in the near-grid region, and electron drift length variation. The extent to which these mechanisms lead to broadening of the alpha-particle peak provides an indication as to their significance in limiting the gamma-ray spectroscopic performance of the coplanar-grid detector. A method by which these mechanisms can be studied is illustrated in Figure 6. The crystal to be analyzed is first fabricated into a coplanar-grid detector using the procedure described in the previous section. The completed detector is mounted into a vacuum chamber and operated as a conventional coplanar-grid detector, while the cathode side of the detector is uniformly illuminated with alpha particles from an ${ }^{241} \mathrm{Am}$ source. This measurement configuration is similar to that used in the previous section in that the detector pulse-height information from the alpha-particle interactions can be used to characterize the electron transport of the material. However, with this coplanar-grid geometry, we are also able to vary both the detector charge induction characteristics and the amount of electron drift length variation, in addition to the detector biases, in order to determine how they impact the resolution of the alpha-particle peak. The adjustment of the charge induction profile is schematically illustrated in Figure $6 \mathrm{~b}$. The method consists of applying a grid bias of sufficient magnitude to ensure complete electron collection at the collecting-grid electrode and then to change the relative gain of the two grid signals prior to subtraction. The charge induction profile of the subtracted signal can be adjusted from that of a simple planar detector $(G=-1)$ to one in which charge induction takes place only in the near-grid region $(G=1)$. The control of the electron drift length variation is shown in Figure 6c. Here, the charge induction profile is first set to that of a planar detector $(G=-1)$. Then the extent of the variation in the electron drift lengths is controlled by adjusting the grid bias, as schematically illustrated in the lower part of Figure 6c. A small drift length variation results when both grids collect the drifting electrons at zero grid bias, whereas the variation is increased to a maximum when the grid bias is large enough to force all of the electron collection to take place at the collecting-grid electrode.

We have performed the above described measurements with a good detector quality, $1 \mathrm{~cm}^{3}, \mathrm{CdZnTe}$ crystal. The effect that the adjustment of $G$ (and consequently, the charge induction profile) has on the alpha-particle spectrum is shown in Figure 7. Typical subtracted preamplifier signals are plotted in Figure 7a for a number of values of $G$. These signals demonstrate the control of the detector charge induction profile from one that increases uniformly throughout the electron drifting process $(G=-1)$ to one that is essentially zero until near the end of the drift process where the induced charge increases rapidly as the electrons drift through the near-grid region $(G=1)$. Shown in Figure $7 \mathrm{~b}$ are the corresponding alphaparticle peaks obtained with the different values of $G$. The peak position is observed to shift to a lower channel number, and the peak width increases as $G$ is adjusted from -1 to 1 . Both these effects lead to the degradation of the alpha-particle peak resolution with increasing $G$ as is shown in Figure 8 where the peak position, FWHM peak width, and peak resolution have been plotted as functions of $G$. The peak shift can be understood based on the following argument. If no electron trapping exists in the detector crystal, a charge $-Q$, drifting from the cathode to the grid electrodes and ultimately collected on the collecting-grid electrode, induces a charge $-Q$ in the collecting-grid measurement circuit and zero charge in that of the noncollecting grid once the charge has been completely collected (see Figure 1). If, however, electron trapping is present, some of the original $-Q$ charge will remain trapped along the trajectory of the dritting charge even after the charge collection at the collecting grid has taken place. The induced charge in the collecting-grid circuit is then equal to the amount of charge physically collected by the collecting grid plus the charge induced by the trapped electrons within the crystal. Additionally, the induced charge in the noncollecting-grid circuit is no longer zero but is equal to the charge induced by these trapped electrons. The adjustment of $G$ is then simply changing the amount of this non-zero noncollecting-grid signal that is subtracted from the collecting-grid signal in order to form the final detector signal. This argument then predicts a linear relation between the peak position and $G$, which is the dependence observed in our measurements (Figure 8a). Furthermore, we have obtained good quantitative agreement between the measured peak position shifts and those predicted with a simple charge induction model that has been described in a previous publication. ${ }^{9}$ An equivalent explanation for the peak shift is 


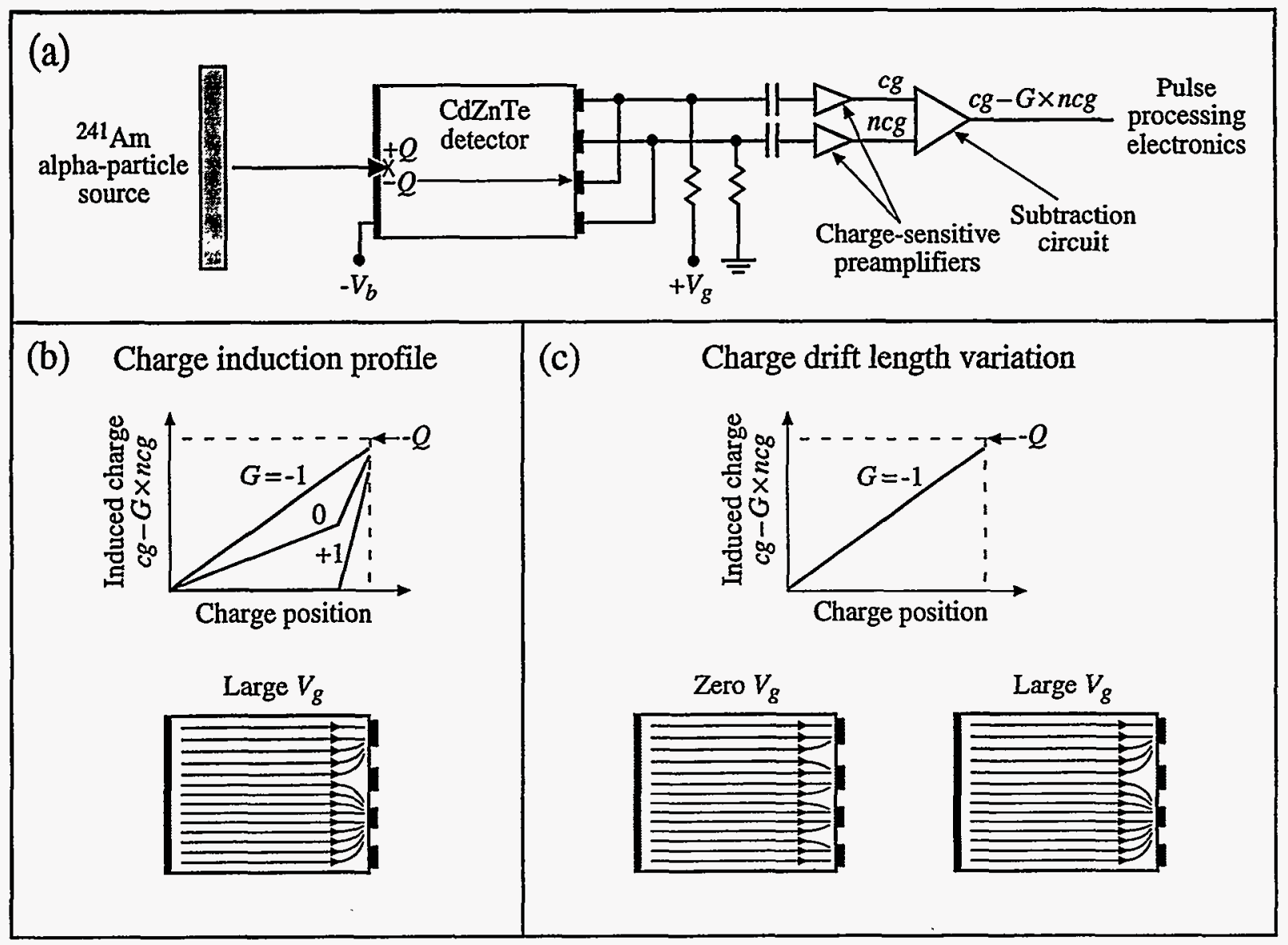

Figure 6. (a) Alpha-particle response measurement configuration for the electron transport uniformity characterization of CdZnTe crystals with a coplanar-grid detector geometry. In this technique an ${ }^{241} \mathrm{Am}$ alpha-particle source is used to uniformly illuminate the entire cathode side of the coplanar-grid detector. Under the influence of the applied bias $V_{b}$, the electrons generated by each alphaparticle interaction near the cathode drift across the entire depth of the detector and are collected by the grid electrodes. The resultant induced charge pulses on the grids are subtracted from each other with a relative gain $G$ to form the detector signal. Any nonuniformity across the detector in the electron transport is reflected as a variation in the pulse heights of the subtracted signal. This measurement configuration can be used to determine the effects of the charge induction profile or electron drift length variation on energy resolution. (b) Schematic illustration of the induced-charge signals (upper picture) and electron trajectories (lower picture) for a method to determine the impact of the charge induction profile on the signal pulse-height variation. By applying a grid bias large enough to ensure complete electron collection at the collecting-grid electrode, the change in the charge induction profile with $G$ can be correlated with the change in the measured pulse-height variation. (c) Similar pictures as in (b) except these illustrate a method to determine the impact of the electron drift length variation in the near-grid region. Here the charge induction profile remains constant by setting $G=-1$, while the electron trajectory variation is controlled with the grid bias $V_{g}$.

that with electron trapping present, the amount of drifting charge resultant from an alpha-particle interaction event decreases along the charge trajectory towards the grids. As $G$ is adjusted from -1 to 1 , the charge induction profile becomes more concentrated near the grids, where the amount of drifting charge for each event is minimum. Consequently, a reduced detector signal results.

The peak width broadening when $G$ is adjusted from -1 to 1 can be understood as a consequence of electron transport nonuniformity. For simplicity, assume that each alpha-particle interaction event produces the same number of electrons. When the charge from a particular event drifts through the crystal to be collected at the grids, the amount of trapping that the charge experiences may differ from that of other events depending on the region of the crystal through which it passes. Therefore, near the cathode there is little variation in charge between each interaction event. However, near the end of the charge drift process, this variation will typically increase as a result of the nonuniformity in the electron trapping. This variation leads to a broadening of the alpha-particle peak width. For the case of $G=1$, the detector signal is completely developed by charge drifting through the near-grid region. Since the variation in the amount of drifting charge between each event will typically be greatest in this region, the $G=1$ case is particularly sensitive to the variation and results in a larger 

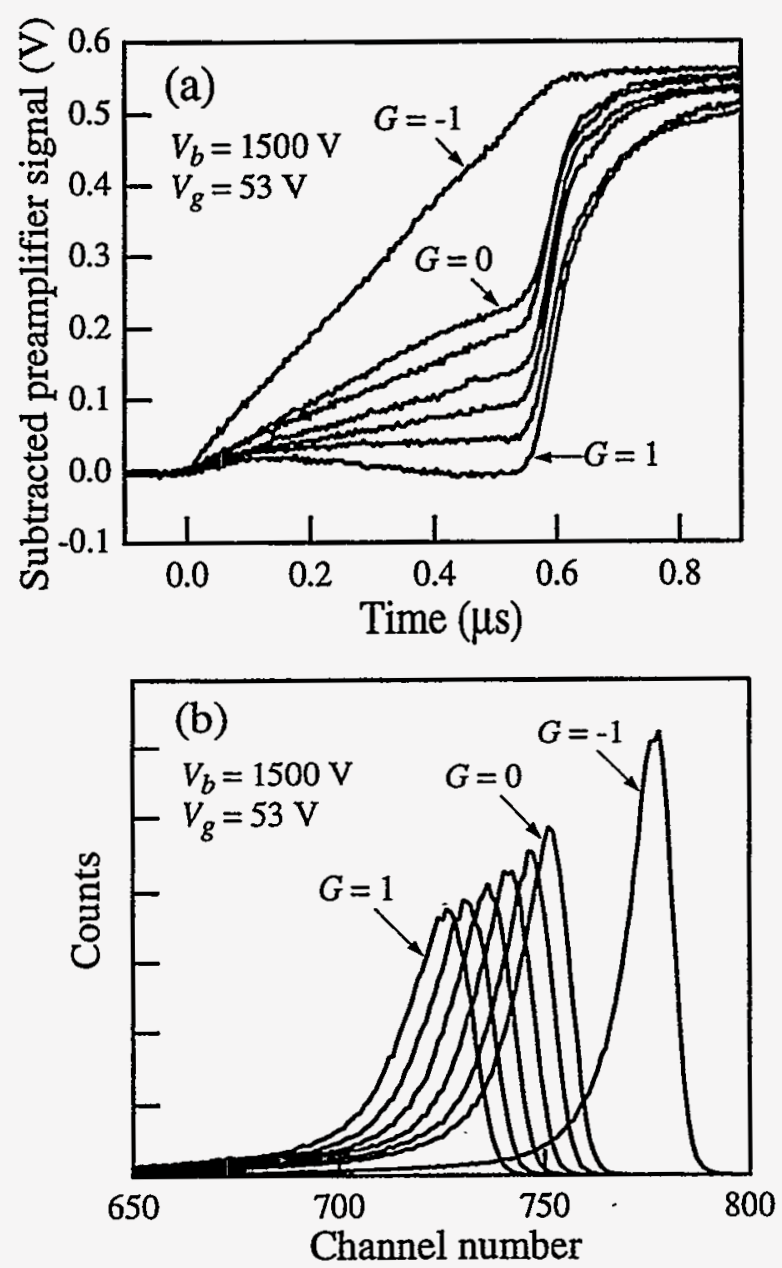

Figure 7. (a) Superimposed differential preamplifier pulse signals, each of which results from the collection of the charge generated by a single alpha-particle interaction event occurring near the cathode of a CdZnTe coplanar-grid detector. The measurement configuration is that described in Figure 6. Each pulse was obtained using a different value of the relative gain $G$. These gain values were $-1,0,0.2,0.4,0.6,0.8$, and 1 , and the detector biases were $V_{b}=1500 \mathrm{~V}$ and $V_{g}=53 \mathrm{~V}$. The detector crystal is $1 \mathrm{~cm}^{3}$ in size and has an electron mobility-lifetime product of about $4 \times 10^{-3} \mathrm{~cm}^{2} /$. (b) Superimposed alpha-particle pulse-height spectra obtained with the same detector and operating conditions used to acquire the data in (a). Only the region of the spectra near the alpha-particle peak has been plotted. The amplifier peaking time used was $8 \mu \mathrm{s}$. Each spectrum was accumulated for the same length of time.
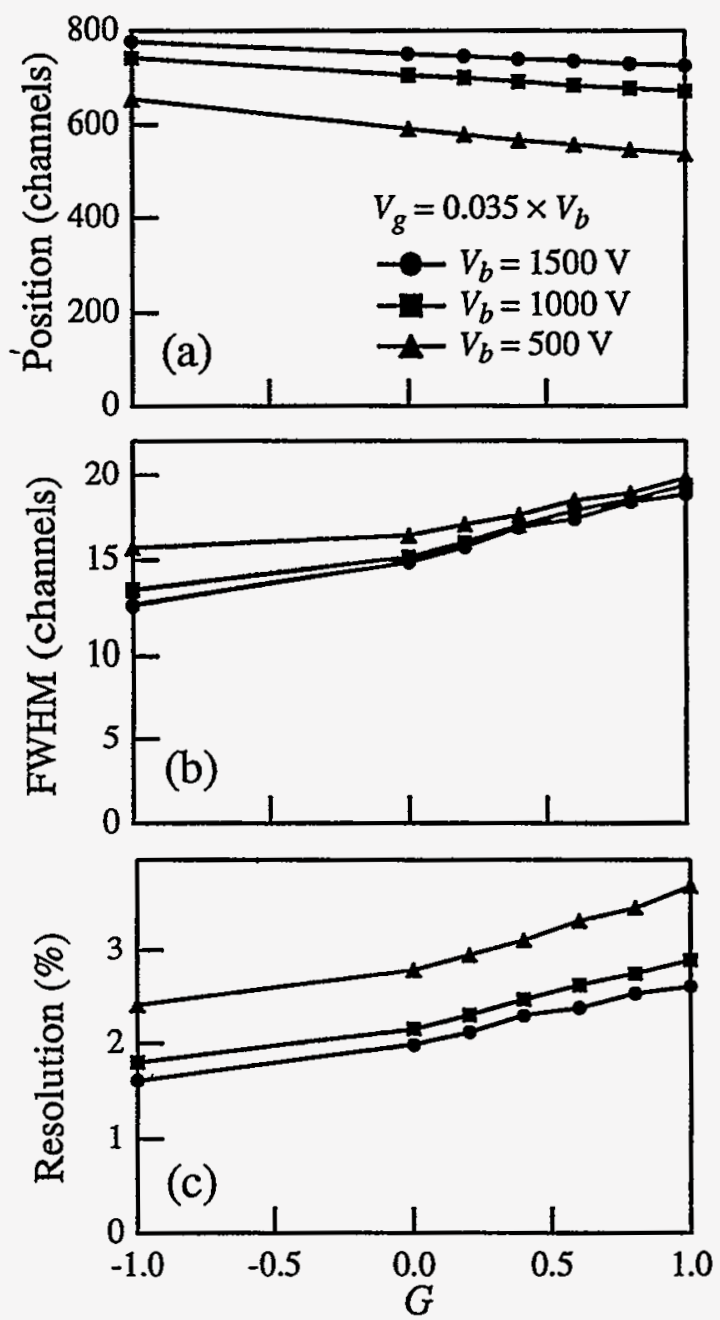

Figure 8. Alpha-particle peak characteristics acquired with the detector and measurement configuration described in Figure 7 plotted as a function of the relative gain $G$. The measurements were obtained using three different cathode biases: $500 \mathrm{~V}$, $1000 \mathrm{~V}$, and $1500 \mathrm{~V}$. The grid bias was adjusted in each case to be 0.035 times the cathode bias. (a) Peak position. This is the channel number within the peak that contains the greatest number of counts. (b) FWHM peak width. (c) Percent FWHM peak resolution.

alpha-particle peak width. In contrast, for the $G=-1$ case, the detector signal is developed uniformly over the entire length of the charge trajectory, leading to a smaller sensitivity to the increased variation in charge near the end of the charge trajectories and, as a result, a smaller peak width.

Plotted in Figure 8 are the results from measurements made at three different detector biases. These measurements illustrate that improved alpha-particle resolution is achieved as the bias applied across the detector is increased. This is due to reduced electron trapping, which increases the peak position and therefore improves the fractional resolution. There also appears to be a small improvement in the absolute peak width at higher bias that may result from a decreased sensitivity to electron transport nonuniformity. In Figure 9 we show ${ }^{137} \mathrm{Cs}$ gamma-ray pulse-height spectra obtained using the bias 


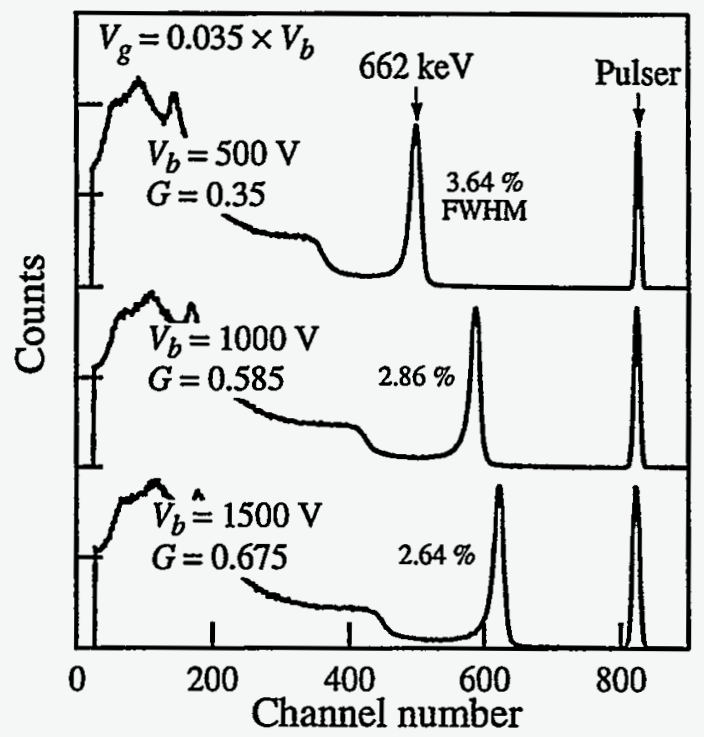

Figure 9. Measured pulse-height spectra of a ${ }^{137} \mathrm{Cs}$ gamma-ray source using the coplanar-grid detector described in Figure 7. A spectrum is shown for each of the bias conditions used to obtain the data shown in Figure 8. The relative gain $G$ was adjusted for each bias condition in order to produce the best possible $662 \mathrm{keV}$ gamma-ray peak resolution. The amplifier peaking time used was $8 \mu \mathrm{s}$. Each spectrum was accumulated for the same length of time.

conditions of Figure 8. Similar to the alpha-particle response measurements, the improved $662 \mathrm{keV}$ gamma-ray resolution at larger detector biases is predominately a result of the decreased electron trapping leading to a larger peak position.

We have also investigated the effect that the grid bias has on electron transport and signal generation using the alpha-particle characterization technique. The results are shown in Figure 10 where the peak resolution and the summed counts within the peak width have been plotted as functions of $G$ for three different grid biases. First note that there is little difference in peak resolution between the two larger grid biases. However, there is a significant degradation in resolution at the lower grid bias of $23 \mathrm{~V}$ for values of $G$ other than -1 . The full-energy peak efficiency as characterized by the counts within the peak width (Figure 10b) also indicates that the low grid bias produces a lower peak efficiency for values of $G$ other than -1 . When the grid signals are added together to form the detector signal $(G=-1)$, there is no degradation in the performance at the low grid bias. This indicates that the charge is collected to the grid surface with the same efficiency regardless of the grid bias. Therefore, the degradation at the low grid bias for values of $G$ other than -1 must be a result of some charge collection outside the collecting grid. For this detector, we then conclude that a grid bias of $23 \mathrm{~V}$ is no longer sufficient to
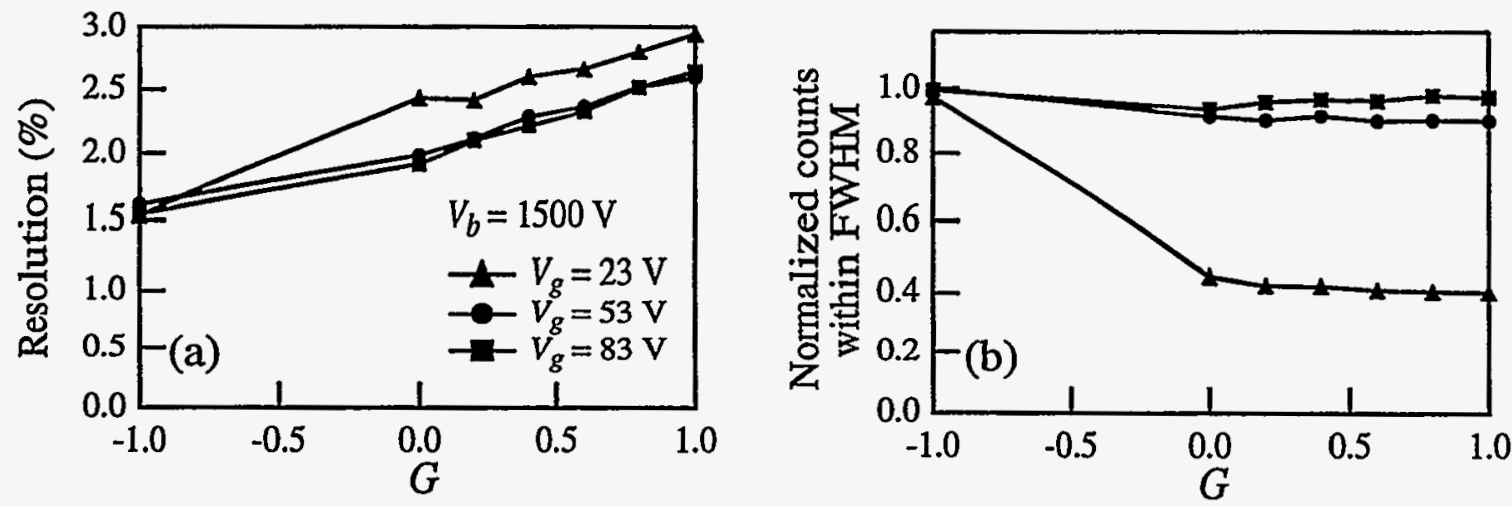

Figure 10. Alpha-particle peak characteristics acquired with the detector and measurement configuration described in Figure 7 plotted as a function of the relative gain $G$. The characteristics have been measured using a fixed cathode bias of $1500 \mathrm{~V}$ and three different grid biases: $23 \mathrm{~V}, 53 \mathrm{~V}$, and $83 \mathrm{~V}$. (a) Percent FWHM peak resolution. (b) Normalized counts within the FWHM peak width. This is the summed counts within the FWHM peak width divided by the largest summed value of any of the measurements. This value characterizes the full-energy peak efficiency. 

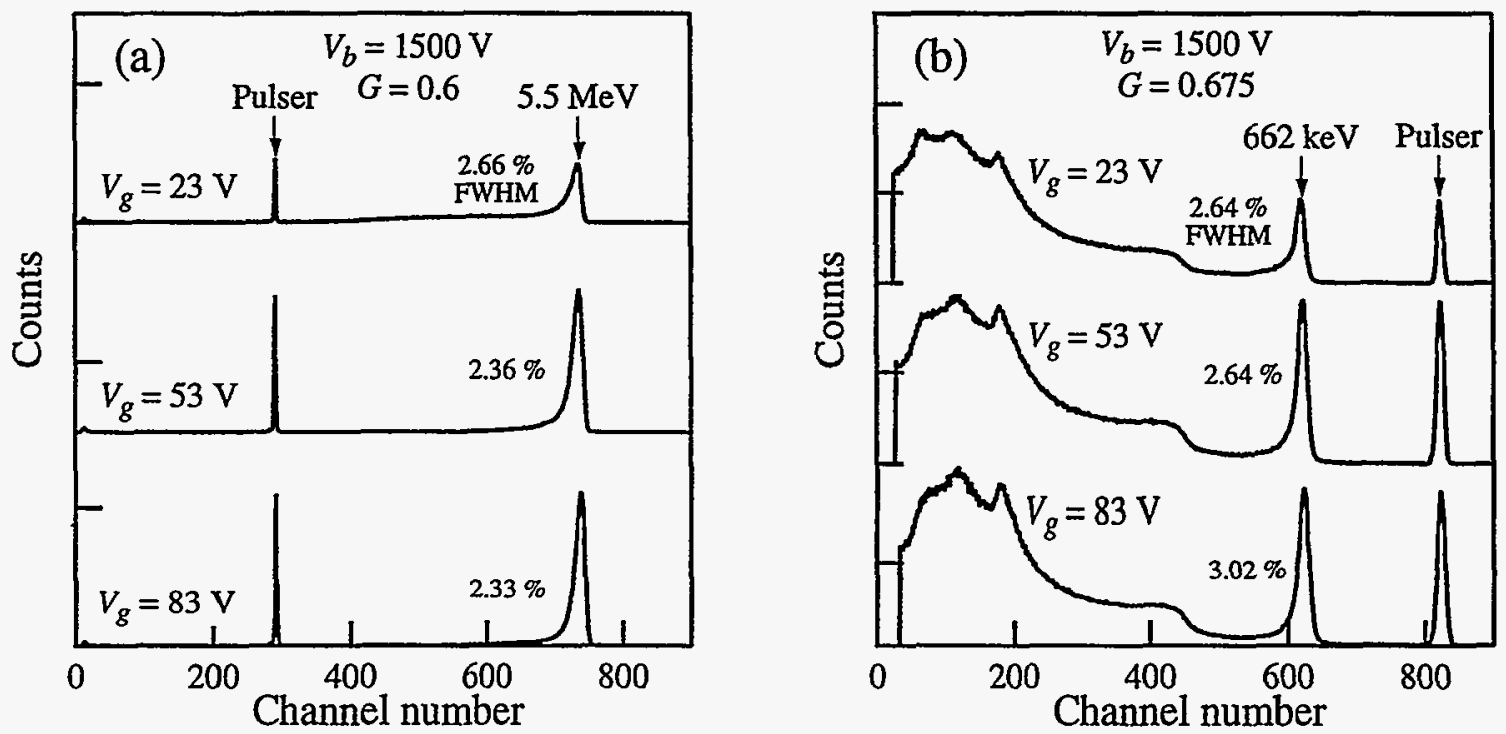

Figure 11. Measured pulse-height spectra using the coplanar-grid detector described in Figure 7. A spectrum is shown for each of the bias conditions used to obtain the data shown in Figure 10. The amplifier peaking time used was $8 \mu$ s. (a) Spectra from an ${ }^{241} \mathrm{Am}$ alpha-particle source. The source was used to illuminate the cathode side of the detector as shown in Figure $6 \mathrm{a}$. The relative gain $G$ was chosen to be similar to that necessary to optimize the gamma-ray detection performance shown in (b). Each spectrum was accumulated for the same length of time. (b) Spectra from a ${ }^{13} \mathrm{Cs}$ gamma-ray source. The relative gain $G$ was adjusted for each bias condition in order to produce the best possible $662 \mathrm{keV}$ gamma-ray peak resolution. Each spectrum was accumulated for the same length of time. The $662 \mathrm{keV}$ gamma-ray FWHM peak resolutions with the electronic noise contributions subtracted are $2.15 \%$, $1.95 \%$, and $2.15 \%$ for the grid biases of $23 \mathrm{~V}, 53 \mathrm{~V}$, and $83 \mathrm{~V}$, respectively.

ensure complete electron collection at the collecting grid, and that some of the charge for certain interaction events is collected by the noncollecting grid or at the gap between the grids.

The $G=-1$ data of Figure 10 also provides information concerning the effect of electron drift length variation on the spectral broadening in this detector. As schematically shown in Figure $6 \mathrm{c}$, the electron drift length variation is largest with a large $V_{g}$ and smallest when $V_{g}=0$. A degradation in the alpha-peak resolution as $V_{g}$ increases from zero would indicate that the drift length variation has a significant impact on the detector performance. As shown in Figure 10, the alpha-peak resolution at $G=-1$ is independent of the grid bias used. Furthermore, the performance was unchanged when the grid bias was set to zero. Therefore, the drift length variation introduced by forcing collection at the collecting grid does not appear to be a significant broadening mechanism in this detector.

Finally, in Figure 11 we compare the ${ }^{241} \mathrm{Am}$ alpha-particle spectra obtained at the grid biases corresponding to those of Figure 10 and the ${ }^{137} \mathrm{Cs}$ gamma-ray spectra acquired using similar operating conditions. As expected from the above discussion, the alpha-particle spectra measured with the two larger grid biases are nearly identical, whereas the small grid bias spectrum has a broadened alpha-particle peak with a substantial low-energy tail that presumably is a result of charge collection outside the collecting grid. With the gamma-ray spectra there is little change in the $662 \mathrm{keV}$ peak resolution among the three grid biases once the electronic noise contribution to the peak width has been subtracted. However, the number of counts within the FWHM peak width of the $V_{g}=23 \mathrm{~V}$ gamma-ray spectrum is approximately half that of those of the higher grid biases. The degradation due to charge collection outside the collecting grid, as identified with the alphaparticle measurements, is apparently reflected in the gamma-ray performance not as a degradation of the $662 \mathrm{keV}$ peak resolution but as a loss of peak efficiency.

\section{SUMMMARY}

To summarize, we have shown examples illustrating that while a CdZnTe crystal with a poor planar-detector alphaparticle response may not necessarily produce a poor gamma-ray energy resolution in a coplanar-grid detector, the poor alphaparticle response does imply that the electron transport of the material is deficient in some way. This deficiency will then lead to a coplanar-grid gamma-ray detector with either degraded resolution, high background, decreased efficiency, or a 
combination of these characteristics. Furthermore, a planar detector alpha-particle pulse-height spectrum with a single sharp peak and little background does indicate that the crystal has the uniform electron transport necessary to achieve good gammaray detection performance with the coplanar-grid technique. This provides a simple method to screen material for coplanargrid detector fabrication.

Alpha-particle response measurements made with coplanar-grid detectors have allowed us to study some of the device-related mechanisms that lead to resolution broadening in the detectors. We have shown that the charge induction profile that leads to an insensitivity to the poor hole transport in the CdZnTe material may also result in an increased sensitivity to any nonuniformity in the electron transport of the material. This sensitivity to the nonuniform transport would typically be greatest when the detector charge induction takes place only for charge drifting through the near-grid region of the detector, as is the case when $G=1$. The $G=1$ operation also produces a smaller pulse height since the charge induction does not take place until after the charge has drifted to the near-grid region and has suffered charge loss from trapping. These measurements, however, have only characterized the detector response- resultant from electron transport. A competing broadening mechanism is the sensitivity to hole transport which plays an increasing role as $G$ is reduced from 1 and could potentially lead to a loss of resolution.

The coplanar-grid alpha-particle response measurements are also useful in analyzing the performance degradation resulting from a reduced grid bias. Based on measurements made as a function of $G$ and as a function of the grid bias, we were able to determine that for our detector and operating conditions, charge collection outside the collecting grid led to the observed performance degradation as the grid bias was reduced. This degradation was manifested as a reduced photo-peak efficiency in the gamma-ray detector response. Finally, based on these measurements, it also appears that the electron drift length variation introduced by forcing collection at the collecting grid did not lead to a significant performance degradation in the tested detector.

\section{ACKNOWLEDGMENTS}

This work was supported by the DOD Office of Special Technology; Technical Support Working Group (TSWG), Project Number T-135.

\section{REFERENCES}

1. H. L. Malm, C. Canali, J. W. Mayer, M-A. Nicolet, K. R. Zanio, and W. Akutagawa, "Gamma-ray spectroscopy with single-carrier collection in high-resistivity semiconductors," Appl. Phys. Lett. 26, pp. 344-346, 1975.

2. R. H. Pehl, N. W. Madden, J. H. Elliott, T. W. Raudorf, R. C. Trammell, and L. S. Darken Jr., "Radiation damage resistance of reverse electrode Ge coaxial detectors," IEEE Trans. Nucl. Sci. 26, pp. 321-323, 1979.

3. P. N. Luke, "Electrode configuration and energy resolution in gamma-ray detectors," Nucl. Instr. Meth. Phys. Res. A 380, pp. 232-237, 1996.

4. H. H. Barrett, J. D. Eskin, and H. B. Barber, "Charge transport in arrays of semiconductor gamma-ray detectors," Phys. Rev. Lett. 75, pp. 156-159, 1995.

5. P. N. Luke, "Single-polarity charge sensing in ionization detectors using coplanar electrodes," Appl. Phys. Lett. 65, pp. 2884-2886, 1994.

6. P. N. Luke and E. E. Eissler, "Performance of CdZnTe coplanar-grid gamma-ray detectors," IEEE Trans. Nucl. Sci. 43, pp. 1481-1486, 1996.

7. Z. He, G. F. Knoll, D. K. Wehe, R. Rojeski, C. H. Mastrangelo, M. Hammig, C. Barrett, and A. Uritani, "1-d position sensitive single carrier semiconductor detectors," Nucl. Instr. Meth. Phys. Res. A 380, pp. 228-231, 1996.

8. Z. He, G. F. Knoll, D. K. Wehe, and J. Miyamoto, "Position-sensitive single carrier CdZnTe detectors," Nucl. Instr. Meth. Phys. Res. A 388, pp. 180-185, 1997.

9. M. Amman and P. N. Luke, "Coplanar-grid detector with single-electrode readout," in Hard X-Ray and Gamma-Ray Detector Physics, Optics, and Applications, R. B. Hoover and F. P. Doty, Editors, Proceedings of SPIE Vol. 3115, pp. 205-213, 1997. 
10. J. R. Heffelfinger, D. L. Medlin, H. Yoon, H. Hermon, and R. B. James, "Analysis of $C_{1-x} Z n_{x} T e$ microstructure," in Hard X-Ray and Gamma-Ray Detector Physics, Optics, and Applications, R. B. Hoover and F. P. Doty, Editors, Proceedings of SPIE Vol. 3115, pp. 40-50, 1997.

11. F. P. Doty, J. P. Cozzatti, and J.P. Schomer, "Characterization of bulk CdZnTe by $\mathbb{R}$ transmission imaging," in Hard X-Ray and Gamma-Ray Detector Physics, Optics, and Applications, R. B. Hoover and F. P. Doty, Editors, Proceedings of SPIE Vol. 3115, pp. 51-55, 1997.

12. P. N. Luke, M. Amman, T.H. Prettyman, P.A. Russo, and D. A. Close, "Electrode design for coplanar-grid detectors," IEEE Trans. Nucl. Sci. 44, pp. 713-720, 1997. 